\title{
Measurements of the Cathode Fall Characteristics in an Electron Beam lonized Discharge
}

\author{
PETER BLETZINGER, SENIOR MEMBER, IEEE
}

\begin{abstract}
Measurements of cathode fall characteristics and of the influence of the cathode fall on the discharge properties of an electron beam ionized discharge (EBD) are reported. Gases investigated included methane, nitrogen, and argon. The cathode fall voltage was found to be a function of discharge current, voltage, gas type and pressure, and the rate of external ionization. At low discharge voltages, the cathode sheath region was found to require microseconds to get established. Added attaching gases had little influence on the cathode fall. Extrapolating to high rates of external ionization, the cathode fall voltage still can be expected to be hundreds of volts for the gases investigated.
\end{abstract}

\section{INTRODUCTION}

$\mathrm{I}$ $\mathrm{N}$ ORDER to operate a gas discharge at higher pressures and over longer periods of time in a diffuse mode, ionization by external means is required. One of the possible sources for this external ionization is a beam of energetic electrons, generally in the energy range of $150-300 \mathrm{keV}$. The electric field applied to the discharge can then be maintained below the selfbreakdown limit. The operating time in the diffuse mode is limited mainly by the power loading into the gas. The voltage applied to the discharge electrodes determines the electric field (or reduced electric field $E / N$ where $N$ is the neutral particle density) in the discharge. Since the gas transport properties are controlled by the reduced electric field, this configuration allows control over specific discharge properties such as electron mobility. The field also determines the electron distribution function (EED) which in turn controls the excitation and population of the energy levels of the discharge gas. This type of discharge has been used for high-pressure electrical discharge lasers. It also has been considered as the switching element in high-speed switch applications, where the external ionization allows both fast switch-on, but even more important, rapid switch-off of high currents [1].

Even though the external source provides the ionization in the volume of the discharge, boundary conditions at the electrodes still have to be fulfilled, for example, the requirements for current continuity at the cathode. Therefore, this discharge will also have a cathode fall in front of the cathode, which can be compared to the cathode fall in a low-pressure self-sustained discharge. Just as in the case of the self-sustained discharge, this cathode fall also can have a major influence on the characteristics of the externally ionized discharge. Especially at low rates of external ionization, the current-voltage characteristics

Manuscript received February 6, 1984; revised March 13, 1984.

The author is the Aero Propulsion Laboratory, Air Force Wright Aeronautical Laboratories, Wright-Patterson Air Force Base, OH 45433 . of an electron beam ionized discharge (EBD) display a very nonlinear behavior, first observed by Velikhov et al. in nitrogen [2]. They also calculated $I-V$ characteristics using the continuity and Poisson equations. This calculation was later refined by Zakharov et al. [3]. In the high-current regime, the cathode fall for the case of a $\mathrm{CO}_{2}$ laser was investigated both theoretically and experimentally by Leland et al. [4]. They found a decrease of the cathode fall voltage with in creasing discharge current similar to the behavior of the cathode fall in the subnormal self-sustained discharge.

For applications of the EBD as a switch, the cathode fall represents part of the voltage loss across the switch. It will be shown that even at high levels of external ionization, this loss still represents a nonnegligible portion of the voltage across the discharge.

To minimize the voltage drop in the main part of the discharge, gases with high electron mobility and therefore high conductivity are of interest. Also, in order to shorten the switch-off time, gases with a large attachment coefficient are added. The measurements being reported here used methane, nitrogen, and argon as the main gases and several added attaching gases. The experimental results will be compared with theoretical calculations and with the characteristics of cathode falls in self-sustained discharges.

\section{EXPERIMENT}

A commercial thermionic cathode electron beam (e-beam) was used in this experiment [5]. The electron energy could be varied from 150-200 keV; an energy of $175 \mathrm{keV}$ was used in these experiments. The e-beam had a cross section of $5 \times 15$ $\mathrm{cm}$ at the foil ( $0.5 \mathrm{mil}$ titanium) and the total beam current in pulsed operation could be varied from less than $1 \mathrm{~mA} / \mathrm{cm}^{2}$ to $25 \mathrm{~mA} / \mathrm{cm}^{2}$. The transmission through the foil was about 40 percent, resulting in e-beam current densities of from $0.4 \mathrm{~mA} /$ $\mathrm{cm}^{2}$ to $10 \mathrm{~mA} / \mathrm{cm}^{2}$ into the discharge volume. The discharge section was part of a very high-vacuum closed-cycle stainless steel flow loop. An externally driven fan provided flow speeds variable to about $4 \mathrm{~m} / \mathrm{s}$ and a heat exchanger in the flow path was used to control the gas temperature. The discharge section used machine glass-ceramic and fused quartz for wall insulation. The anode consisted of a stainless steel grid at a distance of $1 \mathrm{~cm}$ from the e-beam foil. The cathode was made.from solid stainless steel and could be accurately positioned by a micrometer adjustment such that the electrode separation was variable from essentially zero to more than $3 \mathrm{~cm}$. Varying the distance between the discharge electrodes was the method 
used to measure the cathode fall voltage. The discharge was operated in a pulsed mode in order to reach high discharge currents. For lower currents, dc operation was possible. For one particular parameter range, the peak current was held constant and the discharge voltage was measured as a function of electrode spacing. Extrapolating the discharge voltage to zero spacing, the voltage remaining was assumed to be the cathode fall voltage. The contribution of the voltage in the anode fall was neglected, because it is known to be small [3] . Another method to measure field strengths in the EBD discharge volume is to use probes [6]. However, one has to consider effects such as probe shadowing and secondary emission. The biasing of the probes also can become very cumbersome. For these reasons, probes were not used. The current density across the discharge cross section was assumed to be uniform. A check against the current collected by an insulated section of well-defined area in a test cathode verified this assumption. For the pulsed current and voltage measurements transient digitizers with associated data reduction equipment were used.

\section{RESULTS}

As was mentioned above, the cathode fall influences the $I-V$ characteristics of the EBD. This is particularly true for very low amounts of external ionization. To illustrate this, the $I-V$ characteristics of discharges in argon, a mixture of argon and nitrogen, and in pure nitrogen are shown in Fig. 1. All gases were at atmospheric pressure and the discharge and e-beam source were operated with dc. Note that only one of the argon curves was measured with discharge voltage increasing. The nitrogen curves also were measured with the discharge voltage increasing, however only at a few operating points. As derived analytically by Lowke and Davies [7], the current first rises proportional to $V^{1 / 2}$ at a very low rate. At a certain threshold or "ignition voltage," an instability occurs (called a negative differential conductivity by Averin et al. [8]) and the discharge switches to a much higher current at low voltage drop. The voltage was as low as $200 \mathrm{~V}$ for $10 \mathrm{~mA} / \mathrm{cm}^{2}$ discharge current density in argon. If the voltage was subsequently decreased, the unstable region was not encountered, the current decreased monotonically. Particularly interesting is the fact that the argon-nitrogen mixture apparently has a lower conductivity under these conditions than pure argon although it is well known [9] that this mixture has a higher electron drift velocity than pure argon. Similarly, it was found that methane in this operating range has a lower conductivity than that of argon although the electron drift velocity in methane in this range of $E / N$ is at least an order of magnitude larger than that of argon. The reason for these discrepancies is the additional ionization provided by the cathode fall for discharge voltages larger than the "ignition voltage." The $I-V$ characteristics in argon with the high conductivity can be modeled satisfactorily, if two-stage ionization and secondary emission from the cathode are invoked [10].

For a higher discharge current regime, the detailed behavior of the cathode fall voltage for nitrogen and methane at atmospheric pressure is shown in Figs. 2 and 3 for two different e-beam current densities and for cases with an added attaching

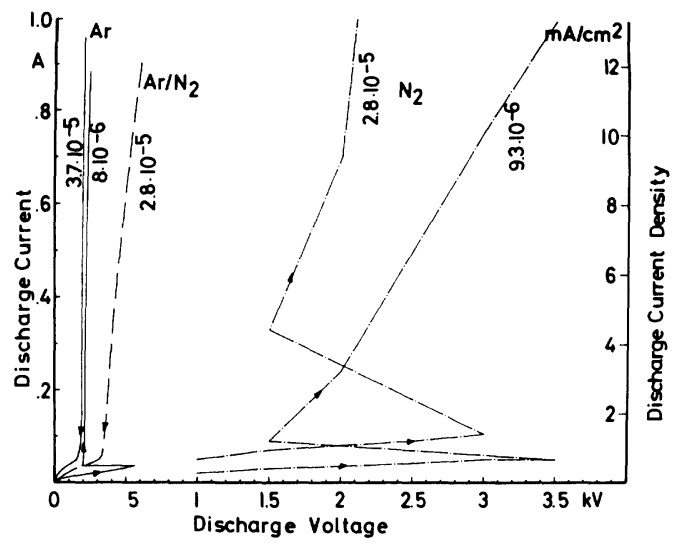

Fig. 1. I- $V$ characteristics of e-beam ionized discharges at very low external ionization rates (dc operation). Characteristics for argon and argon/nitrogen were measured continuously with an $X-Y$ plotter, for nitrogen only a few datapoints per characteristic were measured. The direction of measurement (increasing or decreasing discharge voltage) is indicated with an arrow. All gases are at atmospheric pressure. Curves are labeled with the e-beam current density $\left(\mathrm{A} / \mathrm{cm}^{2}\right)$ used. Argon/nitrogen pressure ratio was $10: 1$, electrode distance $2.2 \mathrm{~cm}$.

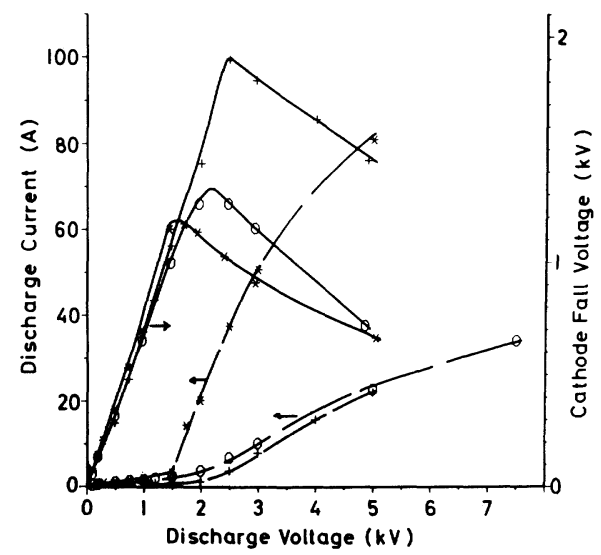

(a)

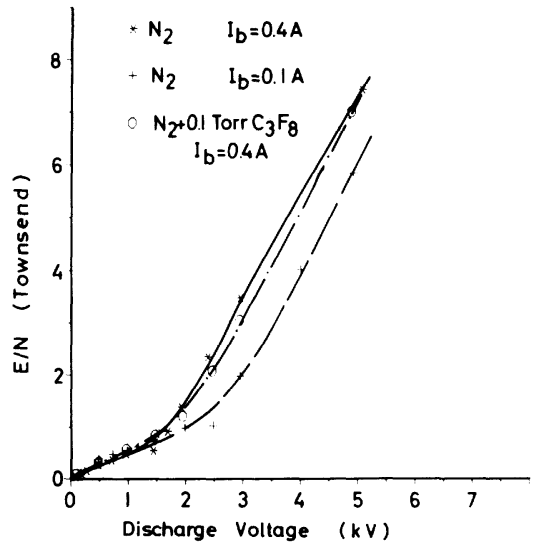

(b)

Fig. 2. (a) Cathode fall voltages (solid curves) and discharge currents (dashed curves) as a function of discharge voltage in nitrogen and nitrogen +0.1 torr of $\mathrm{C}_{3} \mathrm{~F}_{8}, 1$-atm total pressure. E-beam current was 0.4 and $0.1 \mathrm{~A}$ for nitrogen and $0.4 \mathrm{~A}$ for nitrogen $+\mathrm{C}_{3} \mathrm{~F}_{8}$. (b) Reduced electric field for nitrogen and nitrogen +0.1 torr of $\mathrm{C}_{3} \mathrm{~F}_{8}$ as a function of discharge voltage.

gas. Also shown are the discharge currents for the same operating parameters. As in the low-current region, the discharge current at first rises very slowly with discharge voltage up to the "ignition point." For the same range of discharge voltages the cathode fall voltage has a value of about 70 percent of 


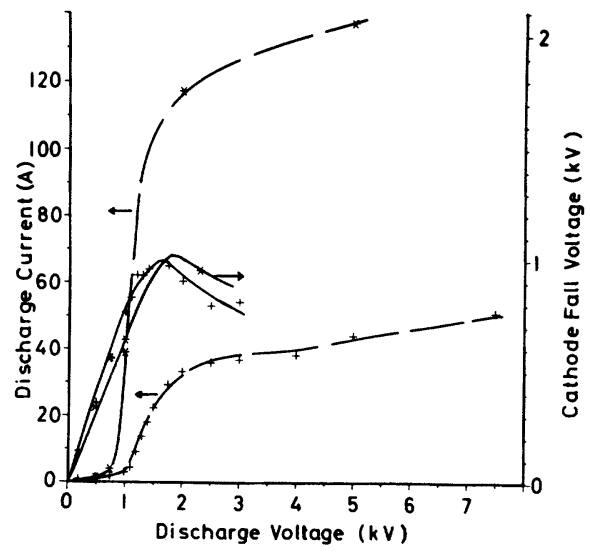

(a)

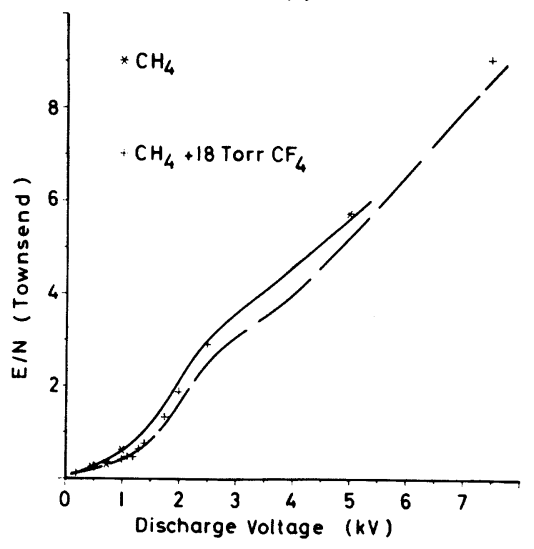

(b)

Fig. 3. (a) Cathode fall voltages (solid curves) and discharge currents (dashed curves) as a function of discharge voltage in methane (atmospheric pressure) and methane +18 torr of $\mathrm{CF}_{4}$. E-beam current was $0.4 \mathrm{~A}$. (b) Reduced electric field for methane and methane + 18 torr of $\mathrm{CF}_{4}$ as a function of discharge voltage.

the total discharge voltage. Beyond the ignition voltage, the cathode fall voltage decreases sharply, having a negative slope similar to the subnormal cathode fall in a self-sustained glow discharge. As the external ionization is increased, the voltage required to reach the high-conductivity regime is reduced. Adding an electron attaching gas will considerably reduce the discharge conductivity in the high-conductivity regime, however the cathode fall voltages are relatively unchanged. This is in agreement with theories for the cathode fall in electronegative gases in self-sustained discharges [11]. The behavior of the reduced electric fields is shown in Figs. 2(b) and 3(b). In the low-current region most of the discharge voltage is taken up by the cathode fall, therefore only in the high-conductivity region can the electric field increase to higher values. The measurements for the methane cathode fall voltages in the high-current region are not as reliable as those for nitrogen. At the higher discharge voltages, the discharge voltage increases faster than linear with increasing distance. This could be caused by the start of Townsend ionization in the volume of the discharge.

As was pointed out in the theoretical study [10], the cathode fall voltage will decrease with increasing external ionization. A measurement of this relationship is shown in Fig. 4 for two different discharge currents for methane at atmospheric pressure. Only at very low e-beam currents does a higher discharge current result in a higher cathode fall voltage. At higher rates

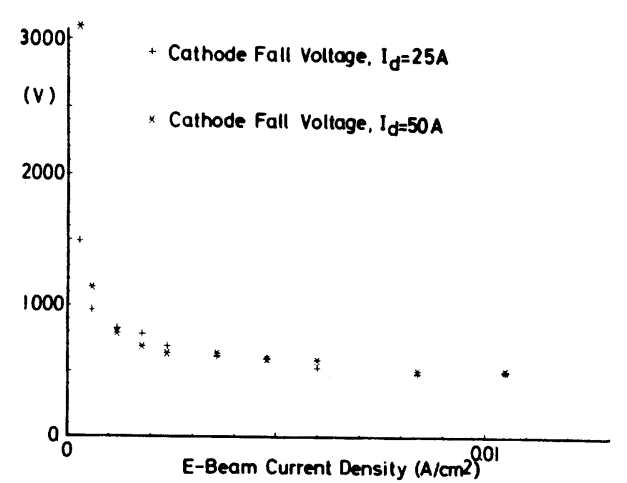

Fig. 4. Cathode fall voltage in atmospheric pressure methane as function of e-beam current density at discharge currents of 25 and $50 \mathrm{~A}$.

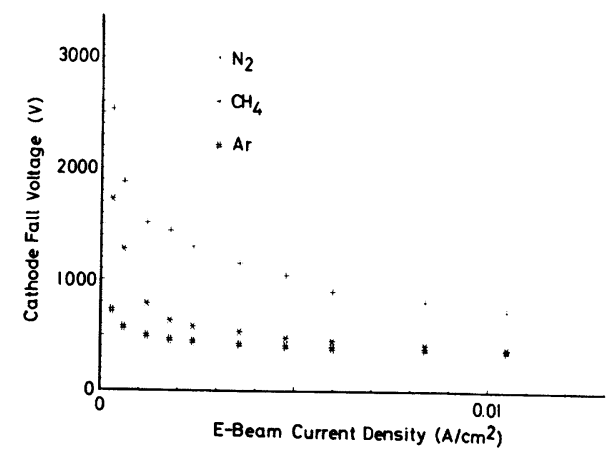

Fig. 5. Cathode fall voltage as a function of e-beam current for nitrogen, methane, and argon at atmospheric pressure.

of ionization, the dependency is below the level of measurement accuracy. The important conclusion from these measurements is that the cathode fall voltage decreases very slowly for higher e-beam currents. Even for very large e-beam currents the cathode fall voltage cannot be reduced below a few hundred volts. As in the self-sustained discharge, the cathode fall also depends on the type of gas used, as shown in Fig. 5 for nitrogen, methane, and argon, all at atmospheric pressure and 0.63 . $\mathrm{A} / \mathrm{cm}^{2}$ discharge current density. As a point of reference, the cathode falls in self-sustained normal low-pressure discharges for a nickel cathode in argon and nitrogen are quoted as 130 and $200 \mathrm{~V}$, respectively [12]. Since the cathode material also influences the cathode fall voltage, an attempt was made to measure cathode fall voltages in materials other than stainless steel. However, tests with a molybdenum cathode, which according to [12] due to its lower work function has a considerably lower cathode fall than either iron or nickel, did not show any systematic decrease of the cathode fall voltage. Considering the vacuum conditions in the test chamber used, this is not too surprising, since even at base pressures in the $10^{-8}$-torr range monolayers of gases build up very rapidly. These gas layers, typically hydrogen and oxygen, can increase the cathode fall by as much as a factor of two and mask the work function of the clean cathode material [13]. Under vacuum conditions normally obtained in larger systems, a decrease of the cathode fall by selection of special materials therefore would seem to be rather difficult to achieve. Note, however, the observations of Velikhov et al. on oxide coated cathodes [2] and of Maslennikov on heated cathodes [14].

The EBD has been modeled with transport coefficients obtained from Boltzmann transport calculations [15]. Consider- 


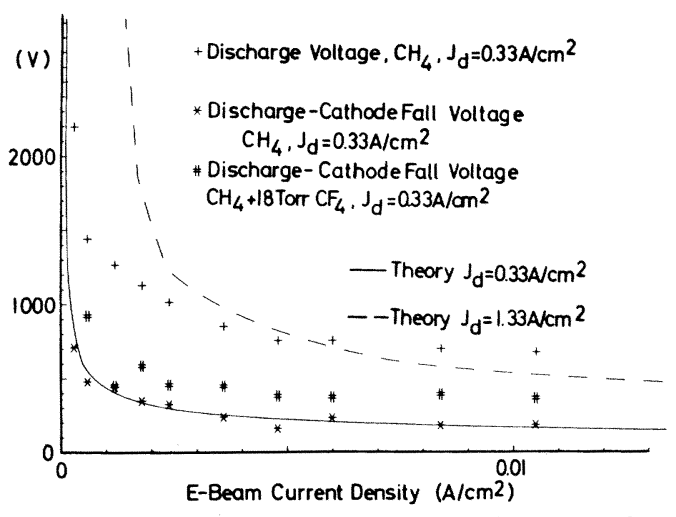

Fig. 6. Comparison of computed discharge voltages as function of e-beam current at two different discharge currents with experimental values for discharge voltages in atmospheric pressure methane. Also shown are experimental values for discharge voltages with the corresponding values for the cathode fall voltages subtracted for methane and methane +18 torr $\mathrm{CF}_{4}$.

ing a methane discharge, which is recombination dominated, one can obtain a relation between source strength, or e-beam current, and discharge voltage. To obtain the discharge current, the electron current density (neglecting the contribution of the ion current) is:

$$
j=n_{e} \cdot e \cdot v_{D}
$$

where $n_{e}$ is the electron density, $e$ is the electron charge, and $v_{D}$ is the electron drift velocity. For the recombination dominated case, the electron density is given by

$$
n_{e}=\sqrt{\frac{S}{\alpha}}
$$

where $S$ is the electron source function (electrons $/ \mathrm{cm}^{3} / \mathrm{s}$ ), $\alpha$ is the recombination coefficient. Then

$$
S=\frac{j^{2}}{e^{2}} \cdot \frac{\alpha}{v_{D}^{2}} .
$$

Both $\alpha$ and $v_{D}$ depend on the reduced electric field and these dependencies are known [15]. The electron source function was obtained from tables [16]. With the discharge current density $j$ as parameter, the e-beam current density required for a particular discharge voltage then can be computed Curves for two discharge current densities are plotted in Fig. 6. The experimental values for the discharge voltage at a corresponding discharge current are seen to be much higher. However, if the contribution of the cathode fall is substrated, a good agreement with the theoretical curve is achieved. A similar series of experimental points is shown for the case of methane with an added attaching gas. Again the contribution of the cathode fall has been subtracted and the increase in the discharge voltage due to the attaching gas is noted. The experimental values for the cathode fall with added attacher were essentially the same as those for pure methane, in agreement with the data in Fig. 2.

Lobanov et al. [17] have presented time dependent calculations of the cathode fall voltages in an EBD and also measurements in nitrogen using probes. In their experiments, the e-beam current was switched on $100 \mu$ s before the discharge current. They noticed a decrease of the cathode fall voltage of about 50 percent over a period of $200 \mu \mathrm{s}$. An example of

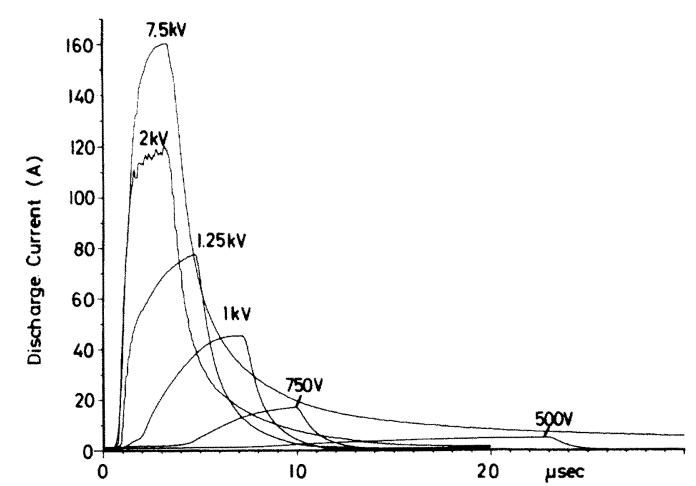

Fig. 7. Time evolution of discharge current in pulsed operation for atmospheric pressure methane. E-beam current was $0.4 \mathrm{~A}$, the parameter is the discharge voltage.

the effect of the dynamic behavior of the cathode fall in methane is shown in Fig. 7. The time for establishing the cathode fall and for entering the high-conductivity region is a function of the applied discharge voltage. As can be noted from Fig. 7, for the lowest discharge voltage of $500 \mathrm{~V}$ it takes more than $20 \mu$ s to reach equilibrium conditions, but for voltages above $2 \mathrm{kV}$ this time is only limited by the switch-on characteristics of the e-beam (the discharge voltage is not switched in this experiment).

\section{CONCLUSIONS}

The cathode fall voltage in a normal low-pressure self-sustained glow discharge depends on the type of gas, pressure, and the material of the cathode. For the subnormal selfsustained glow discharge, the cathode fall voltage in addition is a decreasing function of the discharge current. The cathode fall voltage in the EBD for all the gases measured in the experiments reported here was found to also depend on the type of gas and the discharge current, but in addition it is a function of the discharge voltage and the external rate of ionization. A dependency on cathode material could not be measured for the stainless steel and molybdenum surfaces investigated, however this may be due to surface contaminations, since the very stringent vacuum procedures required for analysis of surface effects could not be followed. In methane, measurements were also performed at a lower pressure (200 torr). At this pressure, the cathode fall was lower by a factor of $2.3 \mathrm{com}$ pared to atmospheric pressure and averaged over e-beam currents from 0.05 to $1.75 \mathrm{~A}$.

Since for switch applications, a minimal cathode fall voltage is desired, the gas should be selected for this characteristic as well as for maximum drift velocity. Fortunately, methane meets both of these criteria. If the switching speed is to be improved by adding attaching gases, the cathode fall voltage, at least for the gases tested, will essentially be unaffected.

Finally, if the EBD is being modeled using available gas transport coefficients and rate data, correction of the theoretical characteristics using the experimentatal values for the cathode fall will result in good agreement between experimental and theoretical characteristics.

\section{ACKNOWLEDGMENT}

The experiments could not have been accomplished without the competent technical assistance of R. Knight. 


\section{REFERENCES}

[1] B. M. Koval'chuk, V. V. Kremnev, G. A. Mesyats, and Yu. F. Potalitsyn, "The injection thyratron-A completely controlled ion device," Sov. Radio. Eng. El. Phys., vol. 21, p. 1513, 1976. R. O. Hunter, "Electron beam controlled switching," in Proc. Int. Pulse Power Conf. (Lubbock, TX), 1976, Pap. IC8.

[2] E. P. Velikhov, S. A. Golubev, Yu. K. Zemtsov, A. F. Pal, I. G. Persiantsev, V. D. Pis'mennyi, and A. T. Rakhimov, "Non-selfsustaining stationary gas discharge induced by electron-beam ionization in N2-CO2 mixtures at atmospheric pressure," Sov. Phys.-JETP, vol. 38, p. 267, Feb. 1974.

[3] V. V. Zakharov, A. A. Karpikov, and E. V. Chekhunov, "Gas discharge in nitrogen with steady-state external ionization," Sov. Phys. Tech. Phys., vol. 21, p. 1074, Sept. 1976.

[4] W. T. Leland, "Relation of electric discharge parameters to shortpulse CO2 laser efficiency," Sov. J. Quant. Electron., vol. 6, p. 466 , Apr. 1976.

W. T. Leland, J. T. Ganley, and M. J. Kircher, "Calculation of cathode fall in electron beam sustained discharges," IEEE Int. Conf. Plasma Science (Austin, TX), 1976.

[5] Energy Sciences, Woburn, MA.

[6] J. H. Jacob, J. P. Reilly, and E. A. Pugh, "Electron-beam spreading and its effect on sustainer current and field distribution in CO2 lasers," J. Appl. Phys., vol. 45, p. 2609, 1974.

[7] J. J. Lowke and D. K. Davies, "Properties of electric discharges sustained by a uniform source of ionization," J. Appl. Phys., vol. 48, p. 4991, Dec. 1977.

[8] A. P. Averin, V. V. Aleksandrov, E. P. Glotov, V. A. Danilychev,
V. N. Koterov, A. M. Soroka, and V.I. Yugov, "Non-self-sustained volume discharges in nonelectronegative gases (theory and experiment)," Sov. Phys. Tech. Phys., vol. 26, p. 665, June 1981.

[9] W. H. Long, W. F. Bailey, and A. Garscadden, "Electron drift velocity in molecular gas-rare gas mixtures," Phys. Rev., vol. 26, p. $665,1976$.

[10] H. Hallada, P. Bletzinger, and W. F. Bailey, "Application of electron-beam ionized discharges to switches-A comparison of experiment with theory," IEEE Trans. Plasma Sci., vol. PS-10, p. 218, 1982.

[11] G. Duke, Thesis, Air Force Institute of Technology, Wright Patterson A.F.B., OH, Dec. 1983.

[12] V. Engel, Ionized Gases, 2nd ed. Oxford, 1965, p. 229.

[13] P. Pich, "Der normale Kathodenfall der Glimmentladung in Neon und die Austrittsarbeit von reinen und gasbedeckten Molybdaenoberflaechen," Beitr. z. Plasma Phys., vol. 7, p. 419, 1967.

[14] N. M. Maslennikov, "Current flow mechanism near the cathode of a non-self-sustaining discharge in nitrogen," Sov. Phys. Tech. Phys., vol. 26, p. 173, 1981.

[15] L.E. Kline, "Performance predictions for electron-beam controlled on/off switches," IEEE Trans. Plasma Sci., vol. PS-10, p. 224, 1982.

[16] L. Pages, E. Bertel, H. Joffre, and L. Sklavenitis, "Energy loss, range, and Bremsstrahlung yield for $10-\mathrm{keV}$ to $100-\mathrm{MeV}$ electrons in various elements and chemical compounds," Atomic Data, vol. 4, p. 1, 1972.

[17] A. N. Lobanov, Ya. I. Londer, L.P. Menakhin, and K. N. Ul'yanov, "Dynamics of the cathode layer in an externally maintained glow discharge," Sov. Phys. Tech. Phys., vol. 24, p. 1204, 1982.

\section{Technical Notes}

\section{A New Method of Protecting Ion Source Accelerators against Deterioration due to Source Breakdown}

M. MATSUOKA, S. MATSUDA, H. NAGAMURA, AND $\mathrm{K}$. WATANABE

\begin{abstract}
A new countermeasure against the surge current due to capacitive stored energy during source breakdown is proposed. The effectiveness is confirmed both numerically and experimentally.
\end{abstract}

Ion sources in neutral beam injectors frequently suffer from beam induced breakdown even after long-term conditioning. Various countermeasures exist to protect ion sources from deterioration due to breakdown [1], [2]. In this note, we propose a new method which is simple, passive and, therefore, reliable.

The applicability of this method has been confirmed in the accel power supply of the prototype NBI for JT-60 [3]. A simplified schematic diagram is shown in Fig. 1. In the figure,

Manuscript received January 3, 1984; revised March 12, 1984.

M. Matsuoka, S. Matsuda, and K. Watanabe are with the Division of Thermonuclear Fusion Research, Fusion Research Center, Japan Atomic Energy Research Institute, Mukai-yama, Naka-gun, Ibaraki-ken, 311-02, Japan.

H. Nagamura is with the Division of Fusion Research, Fusion Research Center, Japan Atomic Energy Research Institute, Mukai-yama, Nakagun, Ibaraki-ken, 311-02, Japan, on leave from the Toshiba Corporation, Japan.

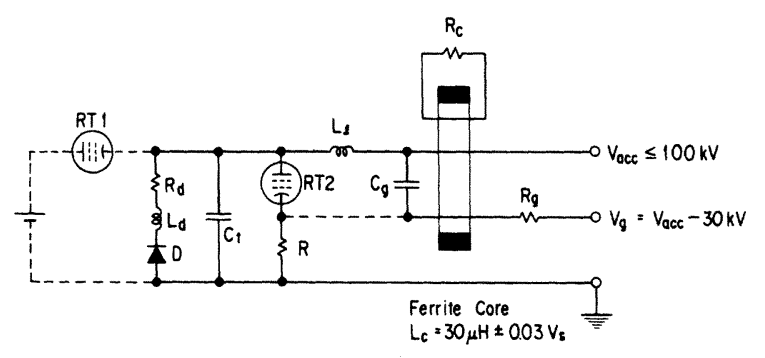

Fig. 1. The schematic diagram of the accel power supply of the prototype NBI for JT-60.

RT1 is a switch tube to cut the output within $20 \mu$ s when source breakdown occurs. The gradient grid voltage $\left(V_{g}\right)$ is supplied by voltage division with the tube RT2 and the resistor $R$. The accel voltage $\left(V_{a c c}\right)$ of up to $100 \mathrm{kV}$ is applied to the plasma grids. $V_{g}$ is normally set to $30 \mathrm{kV}$ lower than $V_{a c c}$. Stray capacitance in this circuit is grouped into two sets. One, which is charged up to $V_{a c c}$, is the sum of the capacitance in the isolation transformer for the plasma generators and the distributed capacitance of the cable duct to the ion sources. This is as much as $24000 \mathrm{pF}$ approximately and is shown as $C_{t}$. The other, which is charged up to $V_{a c c}-V_{g}$, is distributed in the coaxial cable to the plasma grids, whose outer conductor is fixed to $V_{a c c}$ potential. This is about $7000 \mathrm{pF}$ and is shown as $C_{g} . L_{l}$ represents the cable inductance and is about $60 \mu \mathrm{H}$. A ferrite core $L_{c}$ of $30 \mu \mathrm{H}, \pm 0.03 \mathrm{~V} \cdot \mathrm{s}$ with a resistor $R_{c}$ in the secondary winding is present for the surge protection. This protector is equivalent to the parallel connection of $L_{c}$ and 